\section{e0208 NERVE GROWTH FACTOR PROMOTE ANGIOGENESIS OF MESENCHYMAL STEM CELLS}

doi:10.1136/hrt.2010.208967.208

Wang Wenxia, Hu Xinyang, Xie Xiaojie, Liu Xianbao, Wang Jian-an. Second Affiliated Hospital, Zhejiang University College of Medicine, Hangzhou, China

Objective To investigate whether nerve growth factor (NGF) can enhance angiogenesis of mesenchymal stem cells (MSCs), and the possible mechanism.

Methods MSCs were seeded into matrigel-coated 24-well plates, and cultured with NGF at different concentrations $(0 \mathrm{ng} / \mathrm{ml}, 25 \mathrm{ng} / \mathrm{ml}$, $50 \mathrm{ng} / \mathrm{ml}, 100 \mathrm{ng} / \mathrm{ml}, 200 \mathrm{ng} / \mathrm{ml}$ ) for $24 \mathrm{~h}$, the tube formation of MSCs was observed and photographed using an inverted microscope. K-252a, the specific inhibitor of NGF receptor TrkA, was used to inhibit the tube formation promoted by NGF. Western Blot was applied to compare the VEGF expression between different groups. Results NGF can promote MSCs tube formation in vitro, which was peaked at the concentration of $50 \mathrm{ng} / \mathrm{ml}$ with its tubular lengths 2.24-fold increased $(\mathrm{p}<0.05)$, and was attenuated by K-252a. There was no significant difference of the VEGF expression between NGF treated and control group.

Conclusion NGF enhanced the ability of MSCs angiogenesis in vitro, and TrkA signal pathway may be involved.

\section{e0209 PLATELETDERIVED MICROPARTICLES AFFECTS VASCULAR ENDOTHELIAL GROWTH FACTOR EXPRESSION AND THE MECHANISM IN ENDOTHELIAL CELLS}

doi:10.1136/hrt.2010.208967.209

Jia Hongdan, Mao Yongmin, Li Ximing, Cui Rangzhuang, Yu Huining, Pei Chengmin, Cong Hongliang. Tianjin Chest Hospital, Tianjin, China

Objective Detect vascular endothelial growth factor induce by platelet-derived microparticles (PMPs) in human umbilical vein endothelial cells (HUVECs) and its downstream transduction pathway. The aim of our study was to discuss the possible mechanism of PMPs effecting on VEGF releasing and its clinical significance in endothelial cells.

Methods Applying the method of flow cytometry (FCM) to isolate platelet-derived microparticles (PMPs) from platelet poor plasma (PPP), and stained with fluorescin isothiocyanate monoclonal antibody against CD61, applying flow cytometry (FCM) method to detect the quantification of PMPs, and the tolal protein concentration of PMPs was determined using Semi-automatic Biochemical Analyser. HUVECs were commonly cultured. Different concentrations of PMPs intervene HUVECs and incubate for different time. We used semi-quantitative reverse transcription PCR techniques to measure VEGF, phosphatidylinositol-3 kinase (PI3K), extracellular signal-regulated kinase (ERK) and VEGF type II receptor (KDR) mRNA levels.

Results 1. High density of PMPs can be obtained from PPP after activation of ADP, Thrombin and detected by FCM. 2. Different concentration of PMPs intervene HUVECs, VEGF mRNA level in control group (non intervention group) was significantly higher than the four different concentration of PMPs groups $(p<0.05$, respectively). In contrast, KDR mRNA level in control group (non intervention group) was significantly lower than the four different concentration of PMPs groups ( $p<0.05$, respectively). ERK mRNA level in $50 \mu \mathrm{g} / \mathrm{ml}$ PMPs group was significantly higher than the other four groups (1.141 vs $0.749, \mathrm{p}=0.004)$, PI3K mRNA level in $100 \mu \mathrm{g} / \mathrm{ml}$ PMPs group was significantly higher than the other groups (1.344 vs 0.999, $\mathrm{p}=0.004)$. 3. Just as the effect of different concentration PMPs on mRNA expression, VEGF mRNA level in control group (non intervention group) was significantly higher than the $24 \mathrm{~h}$ intervention time group (0.318 vs $0.746, \mathrm{p}<0.001)$. KDR mRNA level in the $4 \mathrm{~h}$ and $24 \mathrm{~h}$ groups were significantly higher than control group ( $<<0.05$, respectively). PI3K mRNA level in $24 \mathrm{~h}$ group was significantly higher than the control group $(2.622$ vs $0.999, \mathrm{p}=0.004)$.

Conclusions Abundant PMPs can be obtained from PPP after activation. PMPs may induce the biological processes of blood vessel and angiogenesis via VEGF and its downstream signal transduction pathways.

\section{e0210 CILOSTAZOL REDUCES NEOINTIMAL HYPERPLASIA BY INHIBITION SUPEROXODE PRODUCTION AND EXPRESSION OF LECTIN-LIKE OXIDISED LDL RECEPTOR-1 AFTER BALLOON COMMON ARTERIAL INJURY IN A RAT MODEL}

doi:10.1136/hrt.2010.208967.210

Hua-ke Su, Yao-ming Song, Shi-yong Yu, Wenyun-Guo, Qiliang-Liu, Wenjun-Li. Department of Cardiology, Xinqiao Hospital, Third Military Medical University, Chongqing, China

Lectin-like oxidised low-density lipoprotein receptor-1 (LOX-1) is a membrane protein that can support the binding, internalisation, and proteolytic degradation of oxidised low-density lipoprotein. The LOX-1 expression and superoxide generation increases in the neointima after balloon injury. Cilostazol, a well-know phosphodiesterase type 3(PDE3) inhibitor for the treatment of peripheral arterial disease, has vasodilator properties and an anti-proliferative action on the growth of vascular smooth muscle cells. To investigate whether cilostazol suppresses intimal hyperplasia and to elucidate its mechanism, we examined the effects of cilostazol to the expression of LOX-1 mRNA and protein, superoxide generation and neointimal hyperplasia of the rat carotid artery after balloon injury. The injury was performed inserting the balloon catheter through the rat common carotid artery and after 14 days a histopathological analysis revealed a significant restenosis with smooth muscle cell proliferation and neointima formation that was associated with an enhanced expression of LOX-1, superoxide generation, Pretreatment of rats with cilostazol $(100 \mathrm{mg} / \mathrm{kg} /$ day $)$ reduced neointima formation, superoxide generation, and LOX-1 expression $(p<0.05)$. Here, we show that Cilostazol reduces neointimal hyperplasia by inhibition superoxode generation and expression of lectin-like oxidised LDL receptor- 1 after balloon common arterial injury in a rat model.

\section{e0211 SI MIAO YONG AN DECOCTION PROMOTES ATHEROSCLEROTIC PLAQUE STABILITY IN VULNERABLE PLAQUE RABBITS}

doi:10.1136/hrt.2010.208967.211

${ }^{1}$ Li Peng, ${ }^{2}$ Jun-Ping Zhang, 'Ming Li, 'Liang-Jun Li, ${ }^{1}$ Ying-Zhi Xu, ' Guang-Yin Zhang, ${ }^{1}$ Cui Yang, ${ }^{1}$ Ya-Nan Zhou. ${ }^{1}$ Central Laboratory of Pharmacology, Tianjin University of Traditional Chinese Medicine, Tianjin, China; ${ }^{2}$ Department of Cardiovascular Medicine, The First Affiliated Hospital of Tianjin University of Traditional Chinese Medicine, Tianiin, China

Background Si Miao Yong An decoction and its medicine Mailuoning injection are proved-effect medicine treating ischaemic cardiovascular disease, but whether it can stabilise the atherosclerotic plaque is currently no clear conclusions. We want to explore the role of Si Miao Yong An decoction interventing on rabbit aortic atherosclerotic plaque instability.

Methods Japanese white rabbits were divided into control group, model group, Simvastatin group and Si Miao Yong An group. The 
animals were killed at the end of the experiment. The pathological changes in aortic was observed by HE colouring, masson staining and oil red staining. CRP, MMP-9, ICAM-1 and NF- $\mathrm{KB} / \mathrm{p} 65$ were tested with ELISA, immunochemistry or RT-PCR.

Results Pathological observation showed that thin fibrous cap and big lipid core area was the feature of plaque in the model group. The main components of plaque were macrophages and fat, collagen and actin content of plaque was little, which had showed the pathological characteristics of unstable plaque. We found atherosclerotic lesions of aorta in two drug groups were lighter than in model group $(p<0.05$ or $p<0.01)$. Si Miao Yong An decoction was superior to Simvastatin in increasing fibrous cap thickness and actin content, reducing lipid core area and MMP-9 expression in plaque $(\mathrm{p}<0.01)$. However, there was no significant difference between the two drugs in reducing NF- $\mathrm{KB} / \mathrm{p} 65$ and ICAM-1 mRNA expression ( $\mathrm{p}>0.05)$.

Conclusions Based on these results, we believe that Si Miao Yong An decoction can promotes atherosclerotic plaque stability by fighting against inflammatory, inhibiting matrix degradation and lipid deposition.

\section{e0212 L-CARNITINE TREATMENT IMPROVES SURVIVAL AND EFFECTS OF TRANSPLANTED BONE MARROW MESENCHYMAL STEM CELLS IN POST-INFARCT RATS HEARTS}

doi:10.1136/hrt.2010.208967.212

Peilei Li, Ming Lin, Changsheng Xu. Department of Cardiology, The First Affiliated Hospital, Fujian Medical University, Fuzhou, Fujian, China

Objective To investigate if the L-carnitine can improve the survival rate of transplanted MSCs after myocardial infarction, and enhance therapeutic effect of MSCs.

Methods Five rats were separately obtained, MSCs were isolated and purified by density-gradient centrifugation and adherence method. 60 rats were randomly assigned into five groups: sham operation group $(n=12)$, model group $(n=12)$, LC group $(n=12)$, MSCs group $(n=12)$, and LC+MSCs group $(n=12)$. Rats in the sham operation group received chest open, without ligation of the left coronary artery. In other four groups, the left coronary artery was ligated to establish myocardial infarction models. Following $20 \mathrm{~min}$ of coronary artery ligation, $250 \mu \mathrm{MSCs}\left(2 \times 10^{6}\right.$ cells per animal) were injected into the left ventricular wall of the infarcted hearts $(50 \mu \mathrm{l}$ into1 injected foci) and peri-infarct zone (200 $\mu$ into 4 injected foci) in MSC group and LC+MSCs group, rats in the model group and sham operation group received intramyocardial injection of the same volume of cell-free DMEM. From 3 days prior to MSC transplantation to ended 4 days post-transplantation, rats in the LC group and LC+MSCs were separately administrated with LC $(100 \mathrm{mg} /(\mathrm{kg} \cdot \mathrm{d}))$ intraperitoneally. Rats in the model group, sham operation group and MSCs group separately administrated with PBS $(100 \mathrm{mg} /(\mathrm{kg} \cdot \mathrm{d}))$. The heart function was evaluated by left ventricular ejection fraction, shortening fraction, and the indexes of blood dynamics 4 weeks after transplantation, the survival of MSCs and myocardial fibrosis in myocardial infarction were detected using immunohistochemistry.

Results Compared with the model group, ejection fraction, fractional shortening, the left ventricular end-systolic pressure (LVESP), left ventricular end-diastolic pressure (LVEDP), the maximal rate of isovolumetric contraction ( $+\mathrm{dp} / \mathrm{dtmax},-\mathrm{dp} / \mathrm{dtmax}$ ) and myocardial fibrosis were improved in the group MSCs, group LC and group LC+MSCs $(p<0.05)$; Compared with the MSCs group, the survival of MSCs, ejection fraction, fractional shortening, LVESP, LVEDP, $+\mathrm{dp} / \mathrm{dtmax},-\mathrm{dp} / \mathrm{dtmax}$ and myocardial fibrosis in group LC + MSCs have significantly improved $(p<0.05)$.

Conclusion Cardiac function and myocardial fibrosis can be improved by LC and MSCs in acute myocardial infarction rat models, but the effect is limited. Pretreated with LC, MSCs transplantation will achieve better result for improving the survival of MSCs.

\section{E0213 COMPARISON OF OLMESARTAN MEDOXOMIL AND CANDESARTAN CILEXETIL ON ANTI-INFLAMMATORY EFFECT IN ATHEROSCLEROTIC RATS}

doi:10.1136/hrt.2010.208967.213

${ }^{1}$ Liang Hui, ${ }^{2}$ Chen Huanzhen. ${ }^{1}$ Dalian Friendship Hospital, Liaoning, China; ${ }^{2}$ Department of Cardiology, The First Hospital Affiliated, China

Objective hs-CRP serum level and the CD40 mRNA expression of aorta in atherosclerotic (AS) rats were measured in this experiment To observe the effect of anti-inflammatory on AS rats treated with olmesartan medoxomil, and further to compare the effect between olmesartan medoxomil and candesartan cilexetil.

Methods 40 male Wistar rats were randomly divided into four groups: A control group, B AS model group, C candesartan cilexetiltreated group, D olmesartan medoxomil-treated group's, C and D groups reproduced AS model, C and D group while giving candesartan cilexetil and olmesartan medoxomil to intervene respectively. 1. $2 \mathrm{ml}$ of blood was collected at 0 week and the end of 8 week, then centrifuged and the supernatant was obtained, hs-CRP serum level was detected with enzyme-linked immunosorbent assay (ELISA), to investigate and compare hs-CRP serum level in each group. 2. To separated the thoracic aorta and abdominal aorta of rats quickly, the expression of CD40 of aorta in every group was detected by reverse transcript polymerse chain reaction (RT-PCR), and finally to take pictures and observe with UV detector. Meanwhile with GADPH as an internal reference agent and conclude the relative expression level of CD40 in aortic tissue, to compare the expression of CD40 in rats aortic among the groups.

Results 1 . At 0 week, the levels of serum hs-CRP were similar among the four groups and showed no significant difference ( $p>0.05$ ); At the end of 8 week, the level of serum hs-CRP in AS rats were significantly higher than that in control group rats $(p<0.01)$, among the two treated groups and no treated group, hs-CRP serum level was reduced obviously in two treated groups $(p<0.01)$, furthermore the level of serum hs-CRP in olmesartan medoxomiltreated group was much lower than candesartan cilexetil $(p<0.01)$. 2. The expression level of CD40 in AS rats aorta was obviously higher than control group $(p<0.01)$, but compared with B group, CD40 mRNA expression in aorta of olmesartan medoxomil and candesartan cilexetil-treated rats decresed significantly $(p<0.01)$, the level expression of CD40 in D group was lower than $C$ group and the difference had statistically significant $(p<0.01)$.

Conclusion 1. The level of serum inflammatory likeas hs-CRP was increased in AS rats, and expressed high level of CD40 in AS rats aorta. 2. Olmesartan medoxomil and candesartan cilexetil can reduce AS rats hs-CRP serum level and decrease the expression level of CD40 in aorta. Both of olmesartan medoxomil and candesartan cilexetil have the effect of anti-inflammatory to AS rats. 3. Compared with candesartan cilexetil-treated, the level of serum hs-CRP and the expression of CD40 decrease in olmesartan medoxomil-treated AS rats, therefore olmesartan medoxomil has a stronger anti-inflammatory effects on AS rats. 